\title{
Avaliação da função pulmonar em diferentes fases da cirurgia de troca de valva cardíaca
}

\author{
Evaluation of pulmonary function in different phases \\ of heart valve replacement surgery
}

Edivânia Soares Silva', Thaís Gouvêa', Jéssica Monteiro Pinto', Thayná Cristinne Oliveira Gomes', Larissa Salgado de Oliveira Rocha ${ }^{2}$, Paulo Eduardo Santos Avila', Rodrigo Santiago Barbosa Rocha ${ }^{3}$

Resumo Objetivo: investigar a função pulmonar em diferentes fases da cirurgia de troca de valva cardíaca. Método: Trata-se de um estudo longitudinal de caráter quantitativo, participaram do estudo voluntários do sexo masculino e com idade entre 25-50 anos, com diagnóstico de valvopatia com indicação cirúrgica de troca de valva cardíaca. Onde foi avaliado o pico de fluxo expiratório (PFE) no período pré-operatório, no período pós operatório na unidade de terapia intensiva (UTI) e no período pós operatório na enfermaria. Foram realizadas até oito medidas, sendo selecionado o maior valor de três medidas reprodutíveis e aceitáveis com variação máxima de $10 \%$ do valor obtido. A análise estatística foi realizada no programa Bioestat 5.0, utilizou-se o teste de Shpiro-Wilk para verificar a normalidade e o teste Anova para análise da variância dos dados, admitiu-se nível de significância $\mathrm{p}<0,05$. Resultados: o pico de fluxo expiratório foi maior na fase pré-operatória $(500,00 \pm 82,15)$ quando comparados ao período de avaliação na unidade de terapia intensiva $(421,11 \pm 46,21)$ e após alta da unidade de terapia intensiva $(452,22 \pm 46,03)$. Conclusão: Foi possível observar alteração da função pulmonar decorrente da cirurgia de troca de valva cardíaca e mesmo após algum tempo decorrido da cirurgia a função pulmonar não retornou aos valores prévios à cirurgia.

Descritores: valva cardíaca; cirurgia cardíaca; função pulmonar.

Summary Purpose: to investigate pulmonary function in different phases of cardiac valve replacement surgery. Methods: This was a longitudinal study of a quantitative nature. The volunteers were male volunteers aged between 25 and 50 years, with valvar heart disease. Where the peak of expiratory flow (PEF) was evaluated in the preoperative period, in the postoperative period in the intensive care unit (ICU) and in the postoperative period in the ward. Up to eight measurements were performed, and the highest value of three reproducible and acceptable measurements was selected with a maximum variation of $10 \%$ of the value obtained. Statistical analysis was performed in the Bioestat 5.0 program, the Shpiro-Wilk test was used to verify normality and the Anova test to analyze the data variance, a significance level of $\mathrm{p}<0.05$ was accepted. Results: peak expiratory flow was higher in the preoperative phase $(500.00 \pm 82.15)$ when compared to the evaluation period in the intensive care unit $(421.11 \pm 46.21)$ and after discharge from the therapy unit (452.22 \pm 46.03$)$. Conclusion: It was possible to observe changes in pulmonary function due to cardiac valve replacement surgery, and even after some time after surgery, pulmonary function did not return to the values prior to surgery.

Keywords: heart valve; cardiac surgery; pulmonary function.

'Universidade da Amazônia - UNAMA, Belém, Pará, Brasil 


\section{Introdução}

A valvopatia pode apresentar-se de maneira congênita ou adquirida, podendo causar estenose valvar com obstrução ao fluxo anterógrado, insuficiência valvar com fluxo retrogrado, ou ambas. Está entre uma das doenças cardíacas mais frequentes na literatura e independente do seu grau de severidade, toda valvopatia ocasiona uma sobrecarga no sistema cardiovascular, que de inicio é considerada tolerada. Porém, com o passar do tempo, essa sobrecarga pode levar a disfunções miocárdicas, gerando a insuficiência cardíaca. Sua incidência apresenta variações, as mais frequentemente encontradas são: cardiopatia isquêmica, valvopatias em adultos e cardiopatias congênitas em idade pediátrica ${ }^{1}$.

Nos casos de cardiopatias crônicas, onde ha um grau alto de acometimento, são indicadas as cirurgias cardíacas. Atualmente, as cirurgias cardiovasculares têm sido bastante utilizadas, devido técnicas aprimoradas e procedimentos cada vez mais seguros, gerando assim resultados positivos ${ }^{2}$.

No entanto, mesmo com o aprimoramento das cirurgias cardíacas, complicações não são descartadas. Pelo fato de ocorrer alterações orgânicas, modificando de diversas formas os mecanismos fisiológicos dos doentes o que implica em cuidados intensivos a fim de estabelecer uma boa recuperação, evitando maiores complicações que podem levar o paciente a óbito. Dentre as possíveis complicações, 58 \% são complicações de maioria pulmonar. As complicações pulmonares mais comuns são: insuficiência respiratória aguda intubação traqueal ou ventilação mecânica por mais de 48 horas após a cirurgia e derrame pleural ${ }^{3}$.

Mediante a presença de doenças cardíacas, ocorre uma diminuição da função pulmonar, além da diminuição da força da musculatura expiratória e da qualidade de vida, facilmente observado na avaliação pré-operatória. No pós-operatório, a dor é um fator influente na diminuição da capacidade pulmonar, pois influência na capacidade do indivíduo de tossir, respirar e realizar movimentos podendo ocasionar pneumonias e atelectasias 4 .

Sendo assim o presente estudo tem como objetivo investigar a função pulmonar de pacientes em diferentes fases da cirurgia de troca de valva cardíaca.

\section{Método}

Trata-se de um estudo longitudinal, de caráter quantitativo, onde participaram da pesquisa 19 voluntários com faixa etária média de $37 \pm 14,1$, do sexo masculino, que foram submetidos à cirurgia de troca de valva cardíaca no Hospital de Clínicas Gaspar Viana no período de março de 2017 à dezembro de 2017, e que se adequaram aos critérios de inclusão e exclusão propostos pelo estudo, sendo excluídos indivíduos que se encontraram na faixa etária menor que 25 anos e maior que 50 anos, que fossem do sexo feminino, em instabilidade hemodinâmica, rebaixamento do nível de consciência, em suporte de oxigênio, que possuísse arritmias cardíacas e que se recusasse a assinar o Termo de Consentimento Livre e Esclarecido.

As avaliações foram realizadas na enfermaria e na Unidade de Terapia Intensiva do Hospital de Clínicas Gaspar Viana após a assinatura de autorização para coleta de dados do atual responsável pela instituição. As avaliações foram aplicadas no período da manhã.

O presente estudo seguiu as normas previstas na resolução 466/12 do CNS, além disso, foi submetido e aprovado pelo Comitê de Ética e Pesquisa do Hospital de Clínicas Gaspar Viana com parecer número 914.702.

Para a coleta de dados, foram utilizados: 1 peakflow, 2 bocais de peakflow, e 1 recipiente contendo glutaraldeído para a higienização dos bocais, sendo este trocado em caso de vencimento do período de utilização.

Para a verificação do pico de fluxo expiratório (PFE), foi utilizado um medidor de fluxo respiratório (Peak Flow Meter) modelo Mini-wright, escala ATS 60-880 L/min, da marca: Clement Clarke ${ }^{\circledR}$ e um clipe nasal durante a realização do teste para garantir ideal vedação.

No momento de utilização, o contador foi colocado à zero, o voluntário se encontrava sentado e o mesmo foi orientado a inspirar o mais profundamente possível, colocar o medidor na boca e apertar a boquilha com os lábios para evitar que o ar se escape para fora do medidor e soprar o mais forte e rapidamente que conseguir, durante 2 segundos. Este procedimento se repetiu por mais duas vezes para que fosse apontado o valor mais elevado das três realizações. Caso o último dos três valores mensurados fosse o maior, havia a necessidade de ser realizada uma nova repetição, até que a última medida não fosse a maior obtida.

A coleta de dados foi realizada em três etapas: a primeira, no período pré-operatório mediante aplicação de uma ficha avaliativa, elaborada pelos pesquisadores e seu orientador, contendo dados pessoais do 
voluntário e avaliação pneumofuncional que foi verificada através de um peakflow; a segunda, no período de 24 horas do pós-operatório por meio de uma reavaliação pneumofuncional do mesmo voluntário; e a terceira no primeiro dia após alta da unidade de terapia intensiva para a comparação dos dados.

Os dados foram processados no software BioEstat versão 5.2, considerando um nível de significância de alfa $=0.05$ para refeição da hipótese nula. Foi realizado inicialmente, para verificar as pressuposições dos testes estatísticos, sendo aplicado para todas as variáveis analisadas, o teste de normalidade Shapiro-Wilk. Em seguida, como os dados contemplaram a metodologia paramétrica, foi utilizado para análise do pico de fluxo expiratório no pré-operatório, na UTI e na enfermaria, o teste ANOVA, para as comparações nas 3 fases.

\section{Resultados}

Participaram do estudo 19 voluntários do sexo masculino, com idade média de $37 \pm 14,1$. Os dados clínicos e demográficos são apresentados na tabela 1.

Os valores obtidos na avaliação do PFE no pré-operatório $(500,00 \pm 82,15)$ demonstraram-se significativamente maiores que os valores encontrados na UTI $(421,11 \pm 46,21)$ com nível de significância 0,01. Quando os valores encontrados no pré-operatório foram comparados aos valores obtidos na enfermaria $(452,22 \pm 46,03)$ também se pode observar que os valores do pré-operatório foram significativamente maiores com nível de significância de 0,01 (Tabela 2).

Quanto à comparação entre a avaliação do pico de fluxo expiratório na UTI $(421,11 \pm 46,21)$ e da enfermaria $(452,22 \pm 46,03)$ pode-se observar que os valores encontrados na enfermaria foram significativamente maiores com nível de significância de 0,01 (Tabela 2).

Tabela 1. Características demográficas e clínicas dos grupos estudados

\begin{tabular}{lc}
\hline \multicolumn{1}{c}{ Variáveis } & GC (n= 19) \\
\hline Idade (anos) & $37 \pm 14,1$ \\
\hline Gênero masculino (n) & $58 \pm 14,6$ \\
\hline FEVE (\%) & $20 \pm 12,2$ \\
\hline Tempo de internação até a cirurgia (dias) & $66,5 \pm 31,9$ \\
\hline Tempo de CEC (minutos) & $18,3 \pm 6,6$ \\
\hline Tempo de VMI (horas) & $8 \pm 1,5$ \\
\hline Presença de infecções (n) & $20 \pm 10,3$ \\
\hline Tempo de internação na UTI (dias) & 20 \\
\hline Alta hospitalar após a cirurgia (dias) & $45,89 \pm 16,60$ \\
\hline Tratamento farmacológico no PO1 & 18 \\
\hline Betabloqueadores (n) & $5,38 \pm 1,49$ \\
\hline Dose (mg) & 38 \\
\hline Anti coagulantes (n) & \\
\hline Dose (mg) &
\end{tabular}

GC: grupo controle; FEVE: fração de ejeção do ventrículo esquerdo; CEC: circulação extracorpórea; VMI: ventilação mecânica invasiva; UTI: unidade de terapia intensiva; PO1: primeiro dia pós-operatório.

Tabela 2. Valores médios, desvio padrão e nível de significância coletados no pré-operatório, UTI e enfermaria de indivíduos submetidos à cirurgia cardíaca de troca de valva. $(\mathrm{p}<0,05)$

\begin{tabular}{ccccc}
\hline & \multicolumn{4}{c}{ MÉDIA \pm DP } \\
& PRÉ & UTI & ENFERMARIA & P-VALOR \\
Período & $500,00 \pm 82,15$ & $421,11 \pm 46,21$ & $452,22 \pm 46,03$ & 0,01 \\
\hline
\end{tabular}

Fonte: dados dos autores, 2018 


\section{Discussão}

O estudo em questão trata da avaliação do PFE antes e após procedimento cirúrgico cardíaco, sendo esta variável analisada um preditor da condição pulmonar intimamente ligada a capacidade de tosse e eliminação de secreções, podendo assim indicar complicações pós-operatórias quando analisada previamente 5 .

Indivíduos cardiopatas tendem a apresentar deterioração da função pulmonar mesmo antes de procedimentos cirúrgicos, configurando assim uma população propensa a maiores complicações pulmonar pós-operatórias ${ }^{6}$. El-Sobkey, Gomaa ${ }^{7}$ foram mais específicos mostrando em sua pesquisa que pacientes submetidos à cirurgia valvar tiveram valores significativamente mais baixos em testes de função pulmonar em relação a outros submetidos à cirurgia de revascularização do miocárdio, evidenciando assim a necessidade de investigar a condição pulmonar nessa população.

As disfunções pulmonares pós-operatórias, podem ser decorrentes de diversos fatores, incluindo as condições pré-operatórias, como presença de comorbidades, tabagismo, gênero e outros ${ }^{8}$. Diante disso Scheeren e Gonçalves ${ }^{9}$, ao avaliarem o pico de fluxo expiratório em pacientes submetidos à cirurgia de andar superior do abdômen observaram que nas 24 horas de pós-operatório o PFE foi menor em relação às 24 horas pré-operatórias, corroborando com a atual pesquisa, sendo esse resultado variável diante de algumas condições, entre elas a homogeneidade da amostra, visto que o sexo masculino o qual compôs totalmente a amostra atual apresentam valores diferenciados de fluxos em relação ao sexo feminino até mesmo antes do procedimento cirúrgico.

Os resultados obtidos no presente estudo vão de encontro à literatura ao evidenciar a diminuição dos volumes pulmonares após procedimentos cirúrgicos, principalmente no pós-operatório imediato ${ }^{10-12}$. Tanto que Cardoso et al. ${ }^{13}$, ao submeterem sua amostra a coleta dos volumes pulmonares e PFE no pré-operatório, pós-operatório imediato e pré-alta hospitalar, constataram que no pós-operatório imediato os pacientes apresentaram menor fluxo expiratório e já os valores coletados no pré-operatório, foram superiores aos demais, estando em concordância com o presente estudo onde os valores coletados após o procedimento cirúrgico foram menores aos que antecederam a cirurgia e os avaliados na UTI (pós-operatório imediato) foram inferiores aos aferidos na enfermaria (pré-alta hospitalar).

Já em pacientes obesos submetidos à cirurgia de gastrectomia, através de uma técnica menos invasiva, o PFE no pós-operatório tardio se mostrou maior ao pós-operatório imediato e até mesmo ao pré-operatório ${ }^{14}$, diferindo-se assim do atual estudo, visto que os valores de fluxo pós procedimento não superaram os valores pré operatórios e a técnica cirúrgica utilizada não configura-se como menos invasiva . Com isso observa-se que a técnica utilizada na cirurgia implicará diretamente nas repercussões pós-cirúrgicas, onde varias literaturas mostram que técnicas minimamente invasivas na troca de valva cardíaca trazem diversos benefícios para o paciente, incluindo a atenuação das complicações pulmonares após a cirurgia ${ }^{15-19}$.

\section{Conclusão}

As complicações pulmonares se mostram iminente as cirurgias cardíacas, sendo seu grau de complexidade dependente das condições pré-operatórias e intra operatórios. Apesar de poucos estudos na literatura, o presente estudo analisou durante as fases de cirurgia cardíaca um pico de fluxo expiratório maior na fase pré-operatória, seguido do período após alta da unidade de terapia intensiva e um PFE menor logo após a cirurgia cardíaca. Sendo sugestivo estudos com acompanhamento prolongado da condição pulmonar.

\section{Referências}

1. Gonçalves SC, Berger SV. Processo Decisório No Manejo De Valvopatias na Insuficiência Cardíaca. Revista da Sociedade de Cardiologia do Rio Grande do Sul. 2004;13(3):1-5.

2. Hernández AA, López HP, Rosales ER, Leyva PEN, Casas ER, Pérez DV. Estatística de pacientes tratados cirurgicamente para valvopatias. Estudo de 36 anos. Rev Cuba Cardiol Cir Cardiovasc. Ano;22(2): x-xx.

3. Soares GM, Ferreira DC, Gonçalves MP, Alves TG, David FL, Henriques KM, et al. Complicações em Cirurgias Cardíacas. Rev.Bras.Cardiol. 2011;24(3):139-46.

4. Medeiros AL, Oliveira AS, Costa SK, Barbosa ML, Oliveira GW. Avaliação da função pulmonar, força muscular respiratória e qualidade de vida no pré-operatório de cirurgia cardíaca. Rev Fisioter S Fun. 2016;5(2):14-22. 
5. Gimenes C, Godoy I, Padovani CR, Gimenes R, Okoshi MP, Okoshi K. Respiratory pressures and expiratory peak flow rate of patients undergoing coronary artery bypass graft surgery. Med Sci Monit. 2012;18(9)

6. Silva JD, Lima CS, Reinaux CM, Brandão DC, Andrade AD. Repercussões da cardiomegalia na função pulmonar de indivíduos adultos com insuficiência cardíaca crônica: uma revisão sistemática. Fisioter Pesqui. 2011;18(1):84-91. http://dx.doi.org/10.1590/S1809-29502011000100015.

7. El-Sobkey SB, Gomaa M. Assessment of pulmonary function tests in cardiac patients. J Saudi Heart Assoc. 2011;23(2):81-6. http://dx.doi. org/10.1016/j.jsha.2011.01.003. PMid:23960642.

8. Fusato HAG, Figueiredo LC, Agostini APRA, Sibinelli M, Dragosava D. Fatores associados à disfunção pulmonar em pacientes revascularizados e com uso de balão. Rev Port Cardiol. 2018;37(1):15-23. http://dx.doi.org/10.1016/j.repc.2017.04.004. PMid:29373223.

9. Scheeren CF, Gonçalves JJ. Avaliação comparativa da função ventilatória através do pico de fluxo expiratório no pré e pós-operatório imediatos de pacientes submetidos a procedimentos cirúrgicos eletivos de andar superior de abdômen. Rev Col Bras Cir. 2016;43(3):165-70. http://dx.doi.org/10.1590/0100-69912016003007. PMid:27556540.

10. Alotaib KD, El-Sobkey SB. Spirometric values and chest pain intensity three days post-operative coronary artery bypass graft surgery. J Saudi Heart Assoc. 2015;27(3):137-43. http://dx.doi.org/10.1016/j.jsha.2015.02.002. PMid:26136627.

11. Baumgasten MC, Garcia GK, Frantzeski MH, Giacomazzi CM, Lagni VB, Dias AS, et al. Pain and pulmonary function in patients submitted to heart surgery via sternotomy. Brazilian Journal of Cardiovascular. 2009;24(4)

12. Urell C, Westerdahl E, Hedenström H, Janson C, Emtner M. Lung Function before and Two Days after Open-Heart Surgery. Crit Care Res Pract. 2012;2012:1-3. http://dx.doi.org/10.1155/2012/291628. PMid:22924127.

13. Cardoso JB, Gass R, Reuter EM, Cardoso DM, Paiva DN. Performance of pulmonary function in patients submitted to cardiac surgery. FIEP Bulletin;2014:84.

14. Ribeiro-Neto NC, Maia LF, Chefer M. Análise Quantitativa da Melhora na Função Pulmonar e da Força Muscular Respiratória entre o Pré e Pós-Operatório de Cirurgia De Gastrectomia Vertical por Videolaparoscopia. Acta Biomed Bras. 2016;7(1):21. http://dx.doi.org/10.18571/ acbm.095.

15. Lamelas I, Sarria A, Santana O, Pineda AM, Lamas GA. Outcomes of Minimally Invasive Valve Surgery Versus Median Sternotomy in Patients Age 75 Years or Greater. Ann Thorac Surg. 2011;91(1):79-84. http://dx.doi.org/10.1016/j.athoracsur.2010.09.019. PMid:21172490.

16. Pineda AM, Santana O, Reyna J, Sarria A, Lamas G, Lamelas J. Outcomes of Reoperative Aortic Valve Replacement via Right Mini-Thoracotomy versus Median Sternotomy. J Heart Valve Dis. 2013;22(1):50-5. PMid:23610989.

17. Santana O, Reyna J, Benjo AM, Lamas GA, Lamelas J. Outcomes of minimally invasive valve surgery in patients with chronic obstructive pulmonary disease. Eur J Cardiothorac Surg. 2012;42(4):648-52. http://dx.doi.org/10.1093/ejcts/ezs098. PMid:22555309.

18. Santana O, Reyna J, Pineda AM, Mihos CG, Elkayam LU, Lamas GA, et al. Outcomes of Minimally Invasive Mitral Valve Surgery in Patients With an Ejection Fraction of 35\% or Less. Innovations:Technology and Techniques in Cardiothoracic and Vascular Surgery. 2013;8(1):1-5. PMid:23571786.

19. Valdez GD, Mihos CG, Santana O, Heimowitz TB, Goldszer R, Lamas GA, et al. Incidence of postoperative acute kidney injury in patients with chronic kidney disease undergoing minimally invasive valve surgery. J Thorac Cardiovasc Surg. 2013;146(6):1488-93. http://dx.doi. org/10.1016/j.jtcvs.2013.06.034. PMid:23972261.

\footnotetext{
Autor correspondente

Rodrigo Santiago Barbosa Rocha

Tv Perebebuí, 2623, Marco

CEP 66087-670, Belém, PA, Brasil

Tel.: (91) 3276-2365

E-mail: rodrigo.santiago.rocha@uepa.br
}

\section{Informação sobre os autores}

ESS, TG, JMP, TCOG são fisioterapeutas graduadas pela Universidade da Amazônia e pesquisadoras.

LSOR é doutora em Ciências do Movimento Humano pela Universidade Metodista de Piracicaba; professora do Centro Universitário do Estado do Pará.

PESA é doutor em Biotecnologia pela Universidade Federal do Pará; professor da Universidade da Amazônia e Fisioterapeuta da Fundação Santa Casa de Misericórdia do Pará.

RSBR é doutor em Ciências do Movimento Humano pela Universidade Metodista de Piracicaba; professor da Universidade do Estado do Pará.

\section{Contribuição dos autores}

ESS: Coleta dos dados e escrita do projeto. TG: Coleta dos dados e escrita do projeto. JMP: coleta dos dados e organização dos resultados. TCOG: coleta dos dados e organização dos resultados. LSOR: revisão dos dados, escrita do artigo científico. PESA: revisão da escrita científica e análise dos dados. RSBR: idealização do tema, orientação do estudo e análise dos dados. 